\title{
Jefferson.
}

\section{Epidermal Growth Factor Receptor Overexpression in Resected Pancreatic Cancer} Amit Mahipal ${ }^{1}$, Mary J. McDonald², Agnieszka Witkiewicz ${ }^{2}$ and Brian I. Carr ${ }^{1}$

${ }^{1}$ Department of Medical Oncology and ${ }^{2}$ Department of Pathology, Thomas Jefferson University, Philadelphia PA

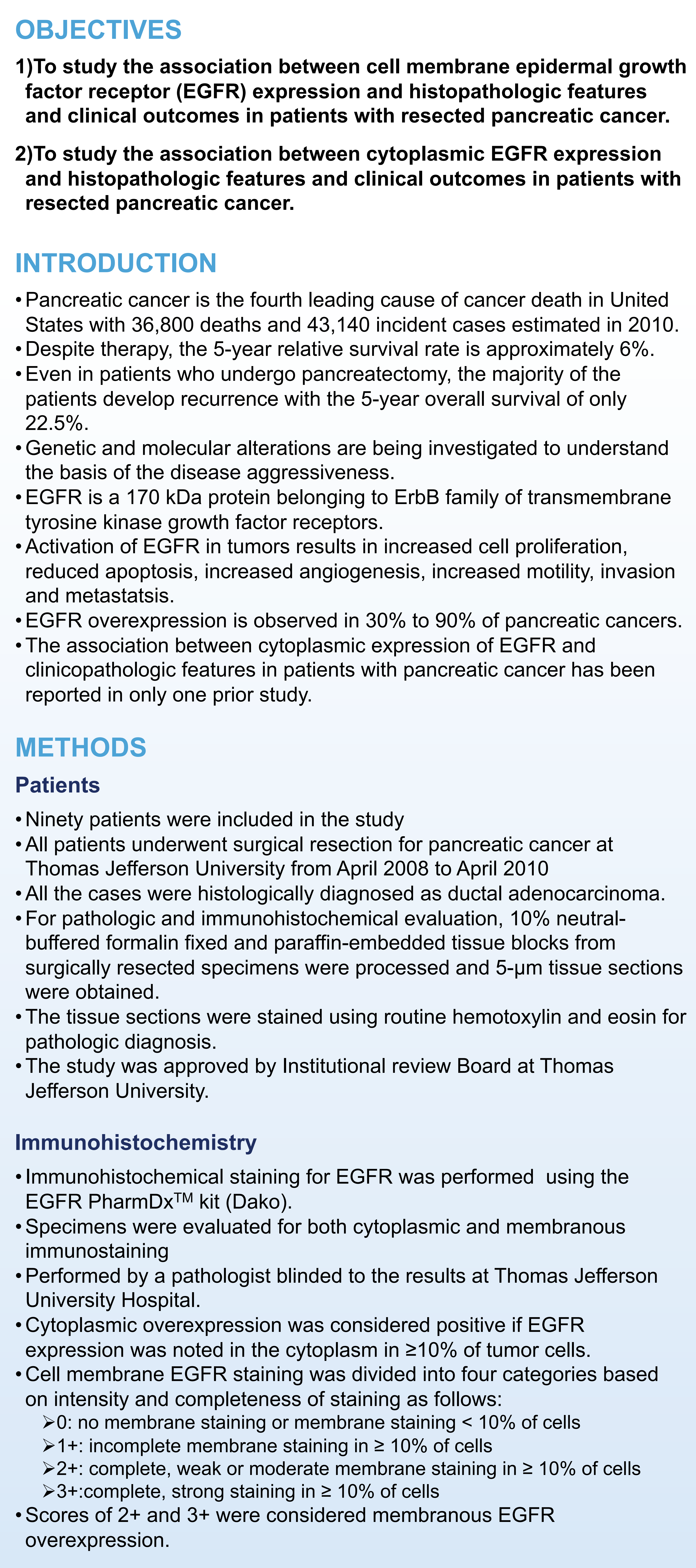

1)To study the association between cell membrane epidermal growth factor receptor (EGFR) expression and histopathologic features and clinical out 2) To study the association between cytoplasmic EGFR expression
and histopathologic features and clinical outcomes in patients with

INTRODUCTION

- Pancreatic cancer is the fourth leading cause of cancer death in United - Despite therapy, the 5-year relative survival rate is approximately $6 \%$ - Even in patients who undergo pancreatectomy, the majority of the patients develop recurrence with the 5 -year overall survival of only $22.5 \%$.
- Genetic and molecular alterations are being investigated to understand the basis of the disease aggressiveness.

ErbB family of transmembrane tyrosine kinase growth factor receptors. Actuation of EGFR in tumors results in increased cell proliferation, and metastattsis, increased anglogenesis, increased motility, invasion - EGFR overexpression is observed in 30\% to $90 \%$ of pancreatic cancers. - The association between cytoplasmic expression of EGFR and clinicopathologic features in patients with pancreatic cancer has been reported in only one prior study.

METHODS

Patients

- Ninety patients were included in the study

- All patients underwent surgical resection for pancreatic cancer at

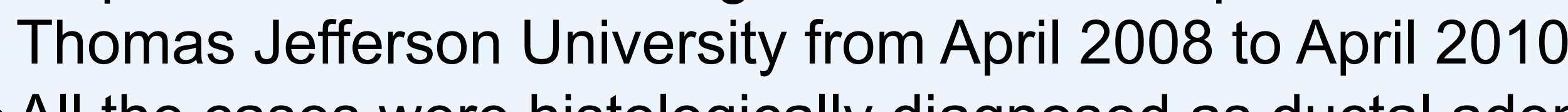

. buffered formalin fixed and paraffin-embedded tissue blocks from surgically resected specimens were processed and $5-\mu m$ tissue sections were obtained.

sections were stained using routine hemotoxylin and eosin for pathologic diagnosis.

- The study was approved by Institutional review Board at Thomas
Jefferson University.

Immunohistochemistry

- Immunohistochemical staining for EGFR was performed using the

-

Performed by a pathologist blinded to the results at Thomas Jefferson University Hospital.

expression was noted in then was considered positive if EGFR

- Cell membrane EGFR staining was divided into four categories based

on intensity and completeness of staining as follows:

. no membrane staining or membrane staining $<10 \%$ of cells

$>2+$ : complete, weak or moderate membrane staining in $\geq 10 \%$ of cells

$>3+$ :complete, strong staining in $\geq 10 \%$ of cells

- Scores of $2+$ and $3+$ were considered membranous EGFR

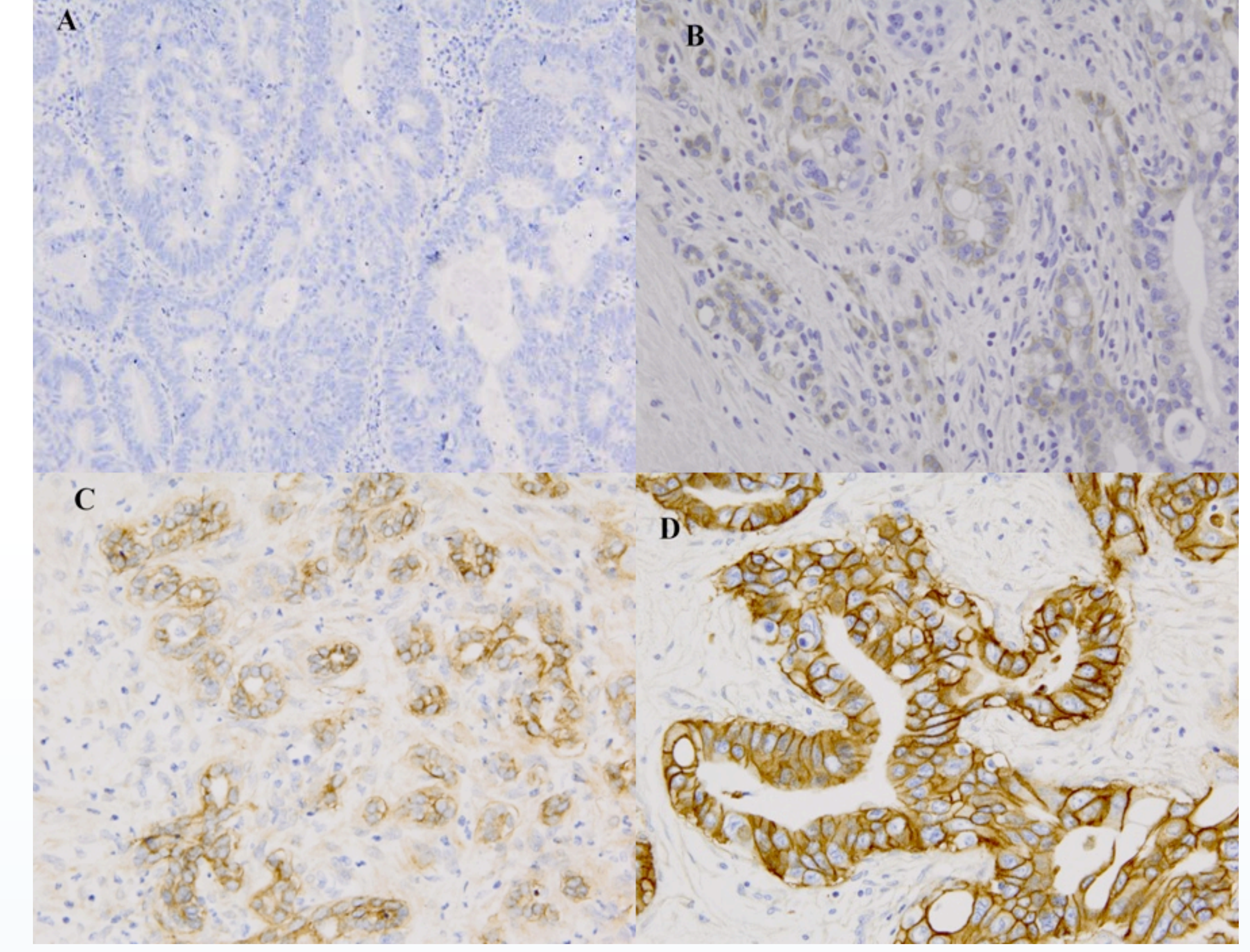

Figure 1. Membrane EGFR expression. A, Score 0; B, Score 1; C, Score 2; D, Score 3

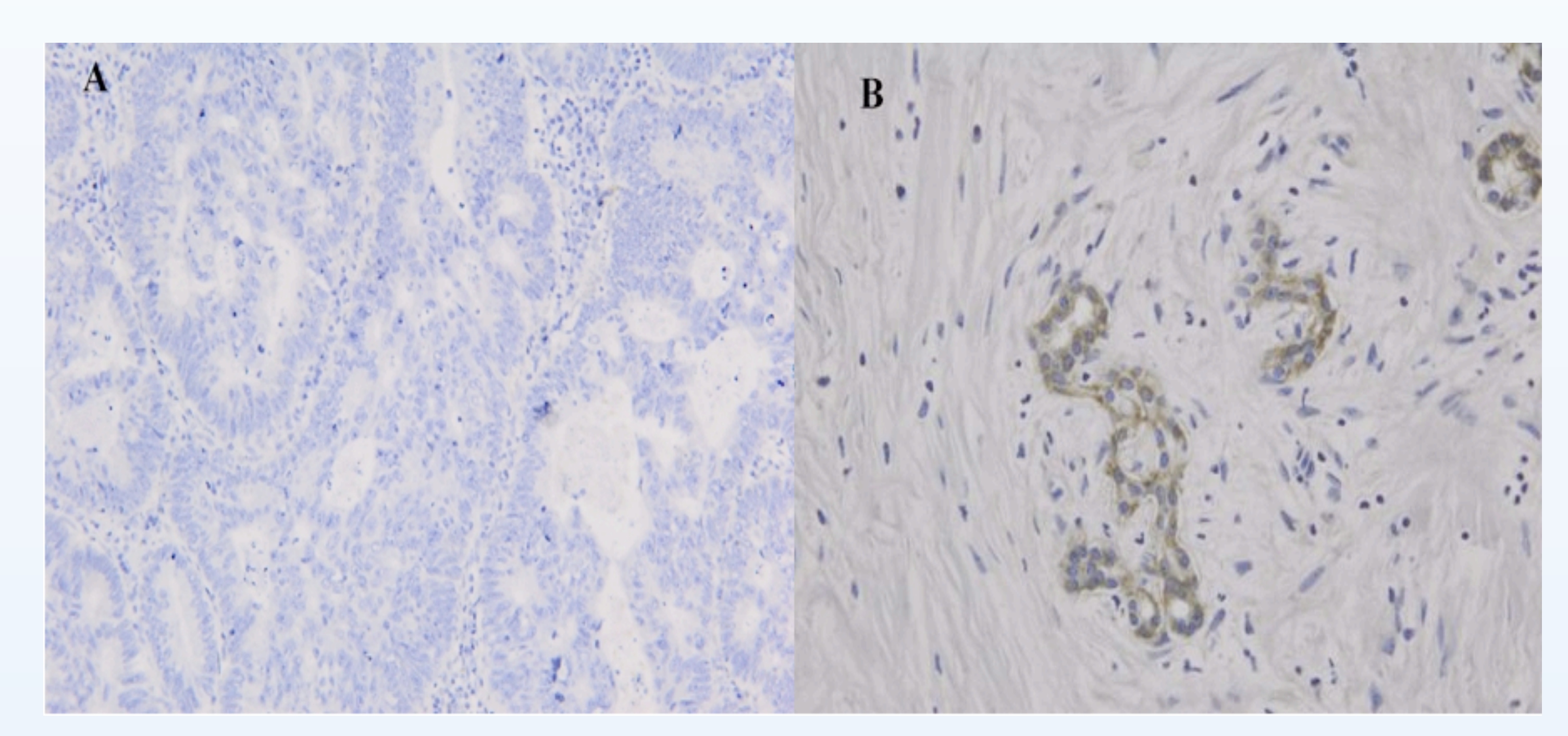

Figure 2. Cytoplasmic EGFR expression. A, negative; B positive

Statistical Analysis

- Descriptive statistics were used to characterize the cohort. Chi-square test was used to determine the association between EGFR overexpression and pathologic features.

Proportional hazard regression analyses were used to estimate - Progression-free survival (PFS) and overall survival (OS) was evaluated using Kaplan-Meier survival curves and differences in survival were lested using log-rank tests.

The ins sided p-value of 0.05 was used for statistical significance. (SAS Institute, Inc, Cary NC).

RESULTS

- 90 patients were included in the study

- The median age was 68 years (Range: 37-92 years)

- Cell membrane EGFR overexpression was observed in 51

- Cytoplamic EGFR overexpression was observed in 64 (71.1\%)

patients

- The median follow up time was 15.5 months

. 52 (58\%) patients had either recurrence or death.

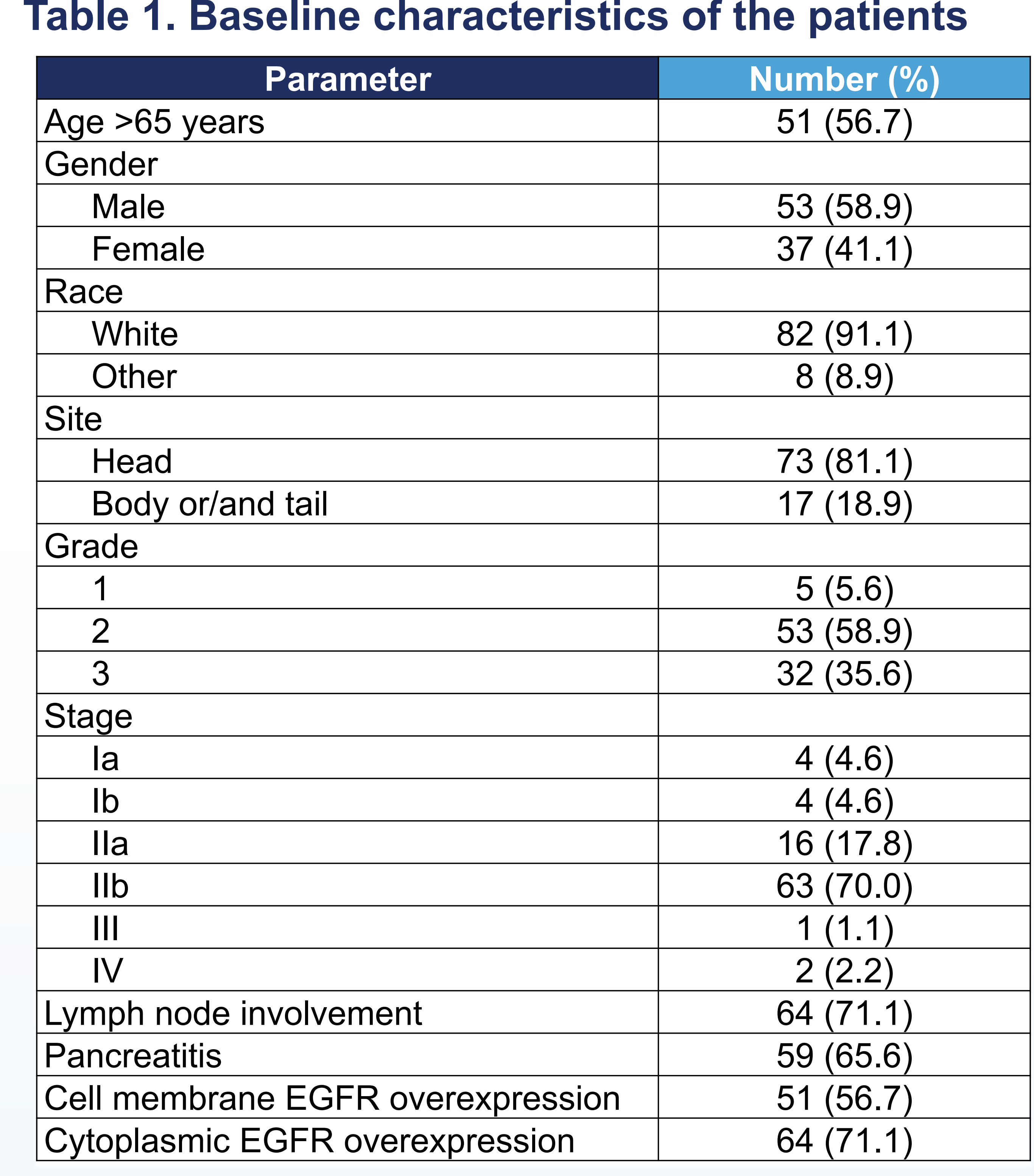

Table 2. Asscociation between cell membrane and cytoplasmic EGFR expression and pathologic features

\begin{tabular}{|c|c|c|c|c|c|c|}
\hline \multirow{2}{*}{ Local invasion } & \multicolumn{3}{|c|}{$\begin{array}{l}\text { Membrane } \\
\text { overexpression } \\
\text { event Absent }\end{array}$} & \multicolumn{3}{|c|}{$\begin{array}{l}\text { Cytoplasmic } \\
\text { overexpression } \\
\text { resent Absent }\end{array}$} \\
\hline & & & & & & \\
\hline yes & 43 & 31 & 0.55 & 52 & 22 & 0.70 \\
\hline & 8 & 8 & & 12 & 4 & \\
\hline Grade 3 & & & 070 & & & \\
\hline $\begin{array}{l}\text { yes } \\
\text { no }\end{array}$ & $\begin{array}{l}19 \\
32\end{array}$ & 26 & & $\begin{array}{l}24 \\
40\end{array}$ & $\begin{array}{l}0 \\
18\end{array}$ & 0.04 \\
\hline Margin positive & & & & & & \\
\hline & 17 & 6 & 0.05 & 22 & 1 & $<0.01$ \\
\hline $\begin{array}{c}\text { no } \\
\text { Vaccular invasion }\end{array}$ & 34 & 33 & & 42 & 25 & \\
\hline $\begin{array}{l}\text { Vascular invasion } \\
\text { ves }\end{array}$ & & & & & & 0.35 \\
\hline $\begin{array}{l}\text { yes } \\
\text { no }\end{array}$ & 27 & $\begin{array}{l}18 \\
21\end{array}$ & 0.52 & $\begin{array}{l}30 \\
34\end{array}$ & 11 & \\
\hline Perineural invasion & & & & & & \\
\hline & 42 & 36 & 0.17 & 57 & 21 & 0.29 \\
\hline & 9 & 3 & & 7 & 5 & \\
\hline $\begin{array}{l}\text { Lymph node } \\
\text { ves }\end{array}$ & 41 & 23 & 0.03 & 42 & 22 & 007 \\
\hline no & 10 & 16 & & 22 & 4 & \\
\hline $\begin{array}{l}\text { Stage Illb or higher } \\
\text { yes }\end{array}$ & 41 & 25 & 0.08 & 44 & 22 & 0.12 \\
\hline no & & 14 & & & & \\
\hline
\end{tabular}

Table 3. EGFR expression and clinical outcomes

Table 3. EGFR expression and clinical outcomes
Progression Free Survival Overall survival

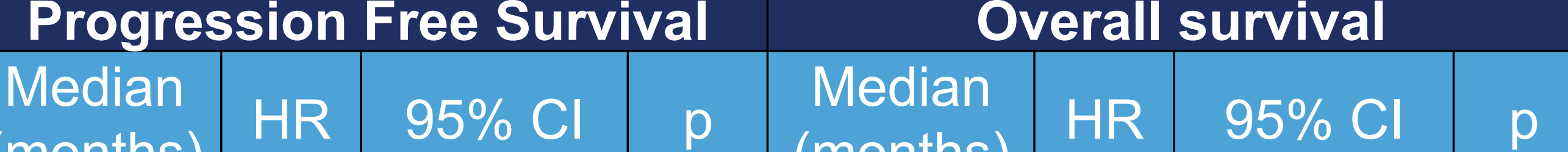
Memb

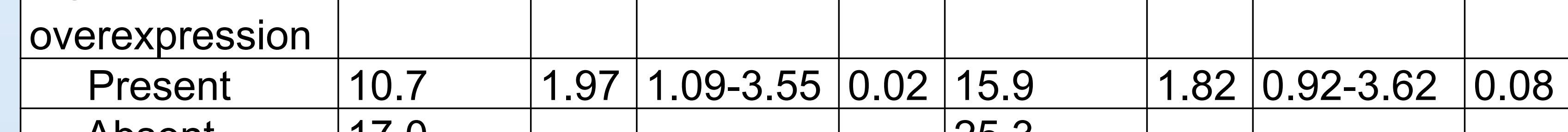
\begin{tabular}{c|c|c|c|c|c|c|c|c|}
\hline Absent & 17.0 & & & & 25.3 & & & \\
\hline $\begin{array}{l}\text { Cytoplasmic } \\
\text { EGFR } \\
\text { Overexpression }\end{array}$ & & & & & & & & \\
\hline Present & 11.7 & 1.39 & $0.74-2.61$ & 0.30 & 17.1 & 1.13 & $0.57-2.27$ & 0.72 \\
\hline
\end{tabular}

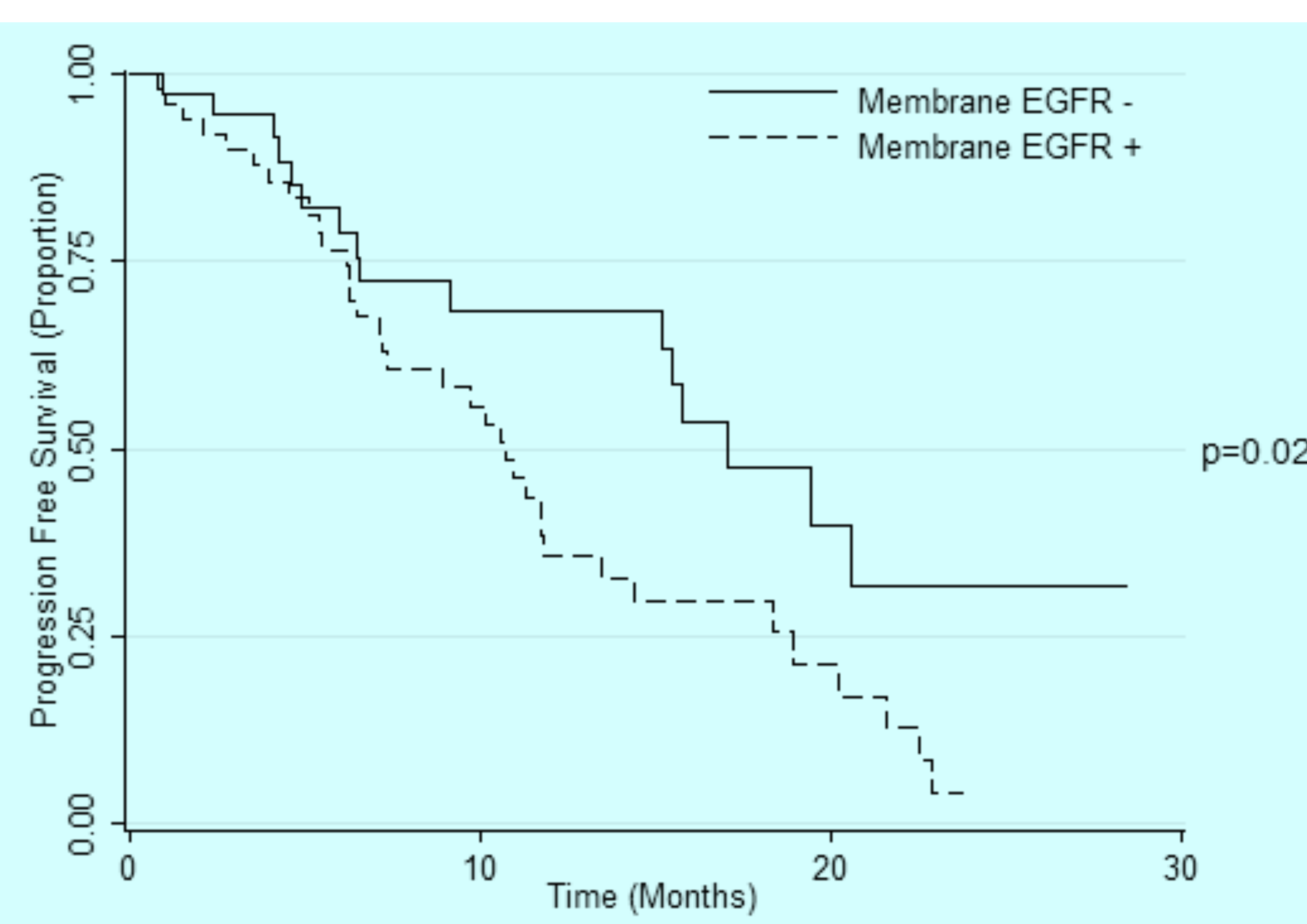

Figure 3. Progression-free survival stratified by cell membrane EGFR expression

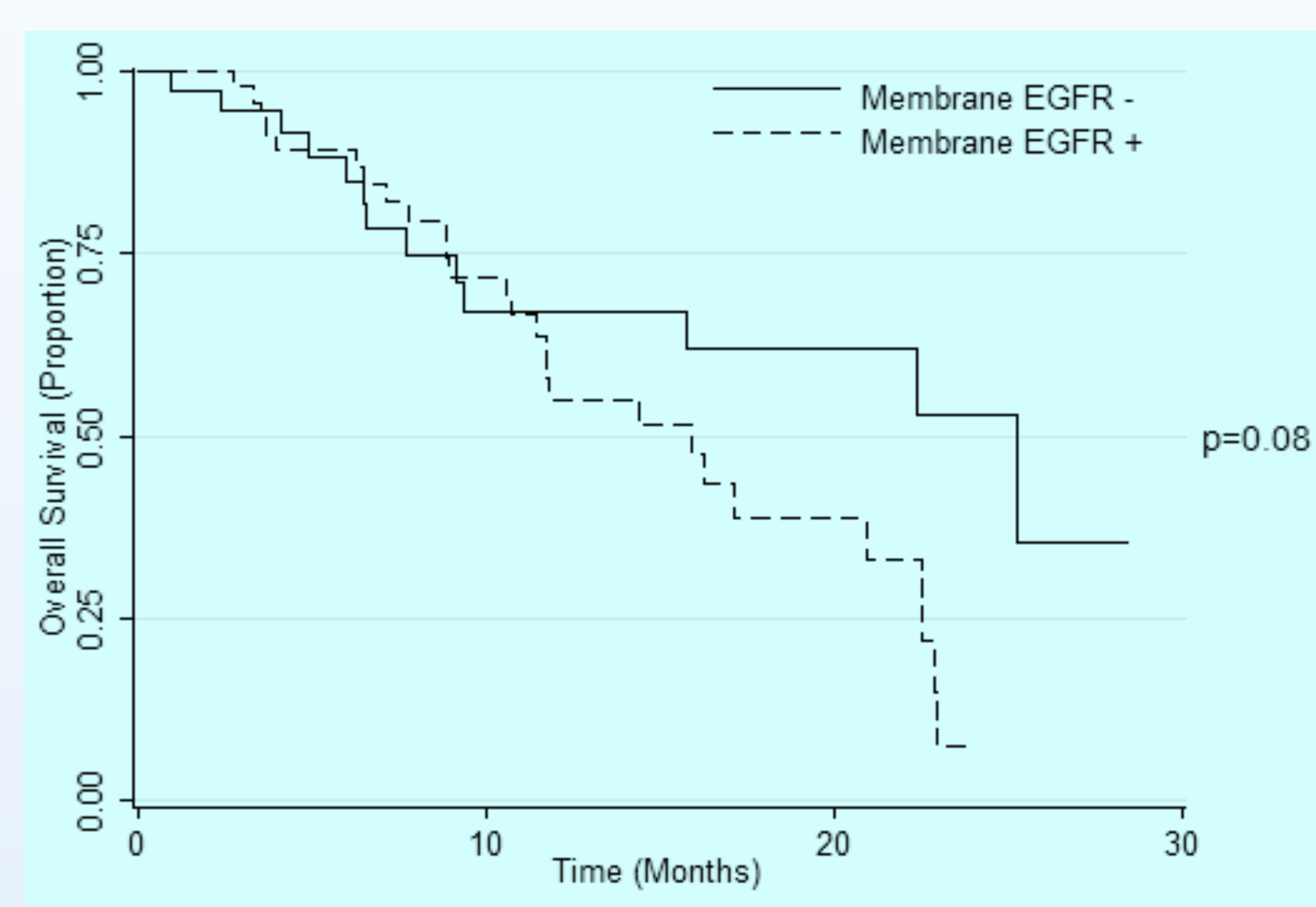

Figure 4. Overall survival stratified by cell membrane EGFR expression

In patients with resected pancreatic cancer:

- Cell membrane EGFR overexpression was associated with lymph

- Cytoplam - In patients with membrane EGFR overexpression, PFS was
significantly shorter (median PFS: 15.9 vs. 23.9 months). There was
no statistically significant difference in survival, although there was a trend towards poorer survival. There was no statistically significan
cytoplasmic EGFR overexpression.

CONCLUSIONS

- Membrane EGFR overexpression is associated with poorer clinical outcomes in patients with

- Cyloplasmic EGFR overexpression is not associated with clinical 